\title{
Evaluation and Comparison of Antioxidant Potential of $\beta$-Glucan from Bacillus Cereus LVK13 with Synthetic Antioxidants
}

\section{Subramaniam Rajendran Yamuna*, Raja Girija, Anandhakumar Brindhalakshmi, Lakshminarayanan Vijayakumar and Sundaramoorthi Pavithra}

\author{
Department of Biotechnology, Bannari Amman Institute of Technology, \\ Sathyamangalam - 638401, India. \\ http://dx.doi.org/10.13005/bbra/2056
}

(Received: 10 February 2016; accepted: 25 March 2016)

\begin{abstract}
$\beta$-Glucan, a homopolymer of glucose which contains mainly $\beta-(1,3)$-d-glucan, was extracted from Bacillus cereus LVK13 using ethanol precipitation. $\beta$-glucan was considerably privileged for its biological importance on comparison with other polymers. As oxidative stress is contemplated to be one of the fundamental causative for various diseases and aging, the antioxidant activity of $\beta$-glucan was investigated to estimate hidden unfamiliar perks associated with $\beta$-glucan in this study. This survey was shaped to compare the scavenging effect of $\beta$-Glucan from Bacillus cereus LVK13 with that of synthetic antioxidants like ascorbic acid and butylated hydroxyl toluene via DPPH $(2,2$ diphenyl-2-picryl hydrazyl) radical scavenging assay. The result of this study showed that isolated $\beta$-Glucan extract of Bacillus cereus LVK13 possesses significant antioxidant properties.
\end{abstract}

Key words: Oxidative stress, $\beta$-Glucan, BHT, DPPH, Bacillus cereus LVK13.

Oxidation, the essential biological processes for production of energy turns unstable primarily by aging and disintegration of physiological functions resulting in stressmediated disorders such as cancer, diabetes, arthritis, asthma, cardiovascular dysfunction, neurodegenerative diseases, etc ${ }^{1,2}$. Hence, for normal functioning of an organism, free radical level maintenance and protection against oxidative stress are indispensable provisions.

Antioxidants as food additive or supplement possess the capability of scavenging reactive oxygen species serving as cell protectors ${ }^{3}$. The hidden mechanism behind their action is the prohibition of preliminary chains responsible for oxidizing reaction. Chemically synthesized

\footnotetext{
* To whom all correspondence should be addressed. Tel: +91-9965573676;

E-mail: yamunasrbtbit@gmail.com
}

butylated hydroxyl toluene (BHT), ethoxyquin malic acid (EMA) and dihydroxy derivatives are widely used commercial antioxidants ${ }^{4}$. Besides, synthetic antioxidants being efficient in radical scavenging, their toxic nature and carcinogenic effects restrict their usage. Hence, it is crucial to interrogate and present efficient harmless antioxidants preferably from natural source.

In seeking for an alternative, $\beta$-glucan produced from Bacillus cereus LVK13 ${ }^{5}$ was found possessing antioxidant property and playing extensive beneficial roles as anti carcinogenic, antiinflammatory, immunomodulatory, antibiotic ${ }^{6,7}$ and anticancer ${ }^{8}$. $\beta$-Glucan, a polymer of glucose is prevalent in foods like oats, barley, wheat, rye, mushrooms, seaweeds, yeasts and in few microbes comprising $\beta$-linked d-glucose molecules. Depending on characteristics of their primary structure, the physicochemical properties of $\beta$ glucan differ significantly $y^{9,10}$. 
Production of $\beta$-glucan from Bacillus cereus LVK13 provides added advantage of economic viability, easier optimization, etc. Hence, this study intended to evaluate the radical scavenging efficiency of $\beta$-glucan from Bacillus cereus LVK13 with synthetic antioxidants like ascorbic acid and BHT by the most widely used 2, 2-diphenyl-2-picryl hydrazyl (DPPH) model system.

\section{METHODSANDMATERIALS}

\section{Production of Crude extract}

Indigenous soil isolate Bacillus cereus LVK13, sub cultured and maintained on nutrient agar slants at $4^{\circ} \mathrm{C}$ was employed for $\beta$-glucan production. Inoculum preparation of Bacillus cereus LVK13 was done in nutrient broth. Further production was carried out in one litre of mineral salt medium supplemented with $10 \mathrm{ml}$ of overnight seed culture and $10 \%$ sucrose, maintained at $30^{\circ} \mathrm{C}$ for 4 days at $180 \mathrm{rpm}$. Subsequent centrifugation of culture at $5000 \mathrm{rpm}$ for 15 min precipitated $\beta$ glucan in ice cold ethanol to produce pellets from where the crude glucan was obtained by lyophilization 5 .

\section{Determination of radical scavenging activity}

Radical scavenging effects of $\beta$-Glucan (BG), Ascorbic acid (AA) and Butylated hydroxyl toluene (BHT) were evaluated by grasping the measure of direct scavenging ability using 2, 2diphenyl-2-picryl hydrazyl (DPPH). The underlying mechanism being generation of free radicals by DPPH on reaction with methanol and grasping of these radicals by antioxidant compounds ${ }^{11}$. Three compounds (BG, AA and BHT) were assayed at different concentrations ranging from $2 \mathrm{mg} / \mathrm{ml}$ to $10 \mathrm{mg} / \mathrm{ml}$ by DPPH for comparison of their antioxidant activity.

\section{DPPH assay}

The three samples at varying concentrations (2-10 $\mathrm{mg} / \mathrm{ml})$ made upto1 $\mathrm{ml}$ with methanol were mixed to $4 \mathrm{ml}$ methanolic solution of DPPH $(0.04 \mathrm{mg} / \mathrm{ml})$ and the mixture was subjected to half an hour incubation in dark. Bleaching of purple color methanolic solution depicted the antioxidant activity of (BG, AA and BHT) the compounds. Absorbances of incubated mixtures were measured against a blank at $517 \mathrm{~nm}$ and percentages of decolorization of the samples were evaluated using the following formula:
Scavenging effect $(\%)=\left(\mathrm{OD}_{\text {control }}-\mathrm{OD}_{\text {sample }} / \mathrm{OD}_{\text {control }} \times 100\right)$ Where, $\mathrm{OD}_{\text {control }}$ represents the absorbance of blank solution (solution with all reagents excluding sample compound) and $\mathrm{OD}_{\text {sample }}$ represents the absorbance of solution containing sample compound. Values of scavenging effect were determined from the graph plotted with scavenging percentage against test compound concentrations ${ }^{12}$.

\section{RESULTS AND DISCUSSION}

DPPH is a stable free radical, which on reaction with reducing agents gets paired-up to attain stability ${ }^{13}$. This nature of DPPH makes it a best choice as substrate for antioxidant activity evaluation. From the noted absorbances, the scavenging effects were calculated for all three compounds and tabulated below (Table 1).

Scavenging effect of $\beta$-glucan from Bacillus cereus LVK13 showed an overall similarity with that of synthetic antioxidants AA and BHT. Among five different concentrations of $\beta$-glucan, the maximum concentration $(10 \mathrm{mg} / \mathrm{ml})$ produced highest percentage of scavenging effect which was about 69. Scavenging activity of $\beta$-glucan at different concentrations was shown in Fig. 1. Nearly a linear pattern of increase was observed, which portrayed direct proportionality of the $\beta$-glucan concentration with scavenging effect.

Ascorbic acid showed scavenging effect of $87 \%$ at minimum concentration $(2 \mathrm{mg} / \mathrm{ml})$ and a highest of $96 \%$ was attained at maximum concentration (10 mg/ml). Fig. 2 clearly revealed the antioxidant efficiency of ascorbic acid as overall scavenging effect was higher than $75 \%$ irrespective of its concentration.

In case of BHT, a steady rise in

Table 1. Scavenging effects of $\beta$-glucan, Ascorbic acid and BHT

\begin{tabular}{lccc}
\hline Concentration & \multicolumn{3}{c}{ Scavenging effect (\%) } \\
\cline { 2 - 4 }$(\mathrm{mg} / \mathrm{ml})$ & $\beta$-glucan & Ascorbic acid & BHT \\
\hline 0 & 0 & 0 & 0 \\
2 & 26 & 87 & 28 \\
4 & 33 & 79 & 49 \\
6 & 57.2 & 75 & 74 \\
8 & 60 & 83 & 82 \\
10 & 69 & 96 & 91 \\
\hline
\end{tabular}


scavenging effect was observed with increase in concentration and a highest of $91 \%$ effectiveness was noticed at $10 \mathrm{mg} / \mathrm{ml}$ concentration (Fig. 3).

All different concentration of $\beta$-glucan from Bacillus sp., however, revealed low radical scavenging effects compared with the synthetic antioxidants. From Fig. 4, it can be clearly seen that among three compounds (BG, AA and BHT), the scavenging effect is higher in ascorbic acid followed by BHT and $\beta$-glucan. Though BG could not attain the maximum, it showed reasonable effectiveness (69\%) on comparison with AA (96\%) and BHT (91\%).

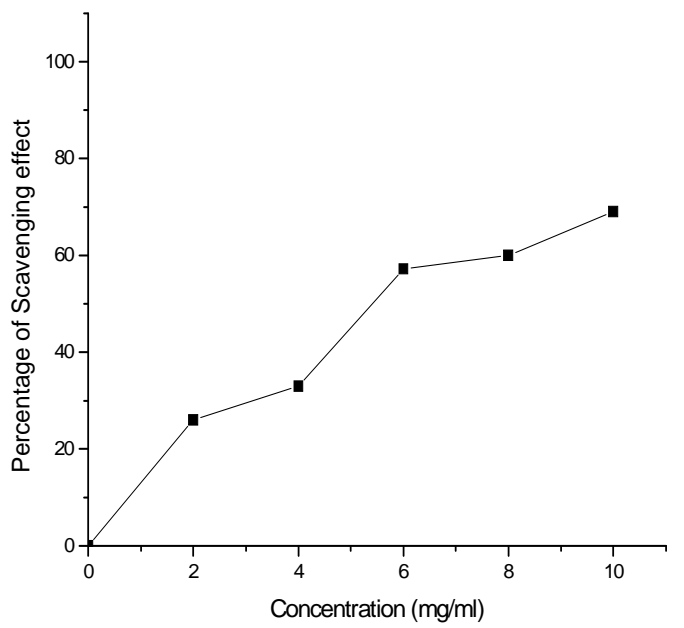

Fig. 1. Scavenging effect of $\beta$-glucan

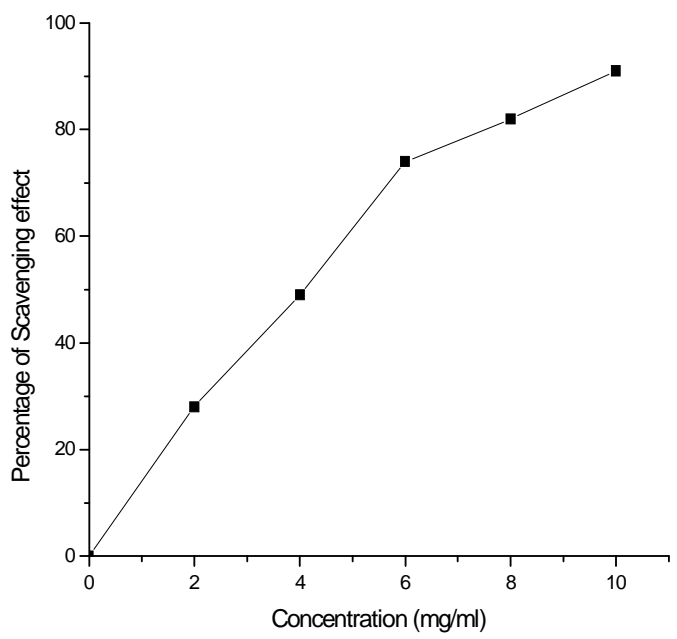

Fig. 3. Scavenging effect of BHT
Synthetic antioxidants prove to be the best radical scavenging agents even at minimum concentration. But the adverse effects caused by them owing to their nature of toxicity and low thermal stability lead to insignificancy of their usage. Hence, replacing synthetic agents with natural ones like $\beta$-glucan proves not only to be suitable substituent but serves multiple purposes enhancing health and beauty. Thus, from the study $\beta$-Glucan from Bacillus cereus LVK13 proved to be a potent alternative for the synthetic antioxidant that causes cancer and other health complications.

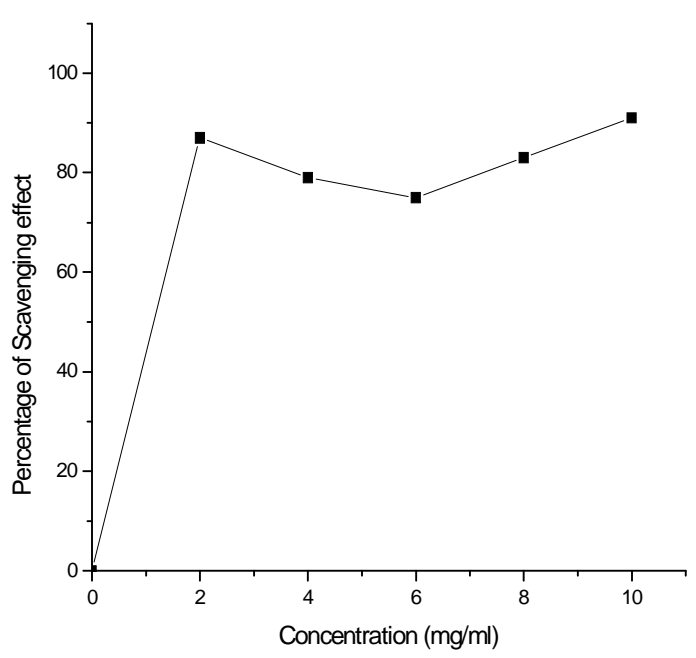

Fig. 2. Scavenging effect of AA

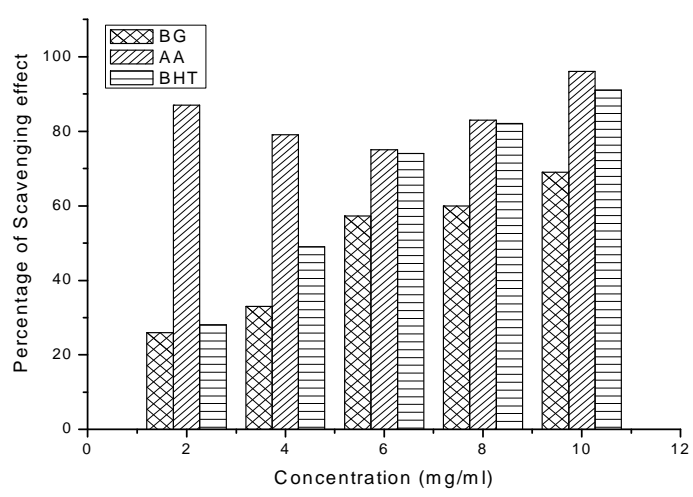

Fig. 4. Comparison of scavenging effect 


\section{REFERENCES}

1. Halliwell, B., Gutteridge, J.M.C. Lipid Peroxidation, Oxygen Radicals, Cell Damage, and Antioxidant Therapy. The Lancet. 1984; 323(8391): 1396-1397.

2. Yagi, K. Lipid peroxides and human disease. Chem. Phys. Lipids., 1987; 45(2-4): 337-351.

3. Yang, J.H., Lin, H.C., Mau, J.L. Antioxidant Properties of Several Commercial Mushrooms. Food Chem., 2002; 77(2): 229-235.

4. Kang, K.A., Chae, S., Lee, K.H., Zhang, R., Jung, M.S., You, H.J., Kim, J.S., Hyun, J.W. Antioxidant Effect of Homogentisic Acid on Hydrogen Peroxide Induced Oxidative Stress in Human Lung Fibroblast Cells. Biotechnol. Bioprocess Eng., 2005; 10(6): 556-563.

5. Vijayakumar, L., Ramalingam, P. Balakrishnaraja, R. Screening, Purification and Characterization of $\beta$-glucan from a novel strain Bacillus cereus LVK13 (KC 898956). Int. J. ChemTech Res. 2015; 8(3): 1156-1162.

6. Asfors, K.E., Ley, K. Sulfated Polysaccharides in Inflammation. J Lab Clin Med., 1993; 121(2): 201-202.

7. Longvah, T., Deosthale, Y.G. Compositional and Nutritional Studies on Edible Wild Mushroom from Northeast India. Food Chem., 1998; 63(3): 331-334.

8. Wasser, S.P. Medicinal Mushrooms as a Source of Antitumor and Immunomodulating Polysaccharides. Appl. Microbiol. Biotechnol., 2002; 60(3): 258-274.

9. Royce, D.J., Bahler, B.D. Bahler, C.C. Enhanced Yield of Shiitake by Saccharide Amendment of the Synthetic Substrate. Appl. Environ. Microbiol., 1990; 56(2): 479-482.

10. Rahayu, A., Al-Shorgani, N.K.N., Hamid, A.A., Yusoff, W.M.W., Daud, F. Optimization of Medium Components Using Response Surface Methodology (RSM) for Mycelium Biomass and Exopolysaccharide Production by Lentinus squarrosulus. ABB., 2013; 4(12): 1079-1085.

11. Cuendet, M., Hostettmann, K., Potterat, O., Dyatmiko, W. Irdiod Glucosidase with Free Radical Scavenging Properties from Fagraea blumei. Helvetica Chimica Acta., 1997; 80(4): 1144-1152.

12. Burits, M., Bucar, F. Antioxidant Activity of Nigella sativa Essential Oil. Phytother. Res., 2000; 14(5): 323-328.

13. Soares, J.R.., Dins, T.C.P., Cunha, A.P., Almeida, L.M. Antioxidant Activity of Some Extracts of Thymuszygis. Free Radic Res., 1997; 26(5): 469478. 